\section{Dentists back ideas to tackle obesity and tooth decay together}

The British Dental Association (BDA) has applauded the new independent report from departing Chief Medical Officer Sally Davies, for offering a joined-up response to the challenges of childhood obesity and tooth decay. ${ }^{1}$

Despite both conditions being driven by common risk factors, they have rarely received a coherent response from policymakers.

The BDA has backed calls for the sweeping measures in the report, including applying VAT to all unhealthy foods, making free drinking water readily available across public places and ensuring that food and drink in nurseries and childcare settings is healthy.

\section{Reference}

Davies S. Time to solve childhood obesity. An Independent Report by the Chief Medical Officer. 2019. Available at: https://assets.publishing.service.gov.uk/ government/uploads/system/uploads/attachment_data/file/837907/cmo-special-report-childhood-obesity-october-2019.pdf (accessed October 2019).

\section{New programme for PLVE candidates}

Led by Leicestershire LDC, the East Midlands Local Dental Committees are piloting a study day programme for Performer List Validation by Experience (PLVE) candidates. This will be open to dentists working in the East Midlands who qualified outside the UK, including EU and non-EU graduates.

All dentists, working within the NHS, are required to be on the national performers' list. The usual route to this is by completing Foundation Training (FT), formerly vocational training, immediately post-qualification.

Dentists qualifying outside the UK may not be eligible for Foundation Training and need to obtain a performer number, through the PLVE programme. The applying dentist has to be assessed by NHS England in consultation with Health Education England (HEE) to decide whether they can be admitted to the list. In many respects PLVE mirrors the FD process.

Both FT and PLVE require the dentist seeking the performer number to work under the supervision of an approved trainer/ mentor in an approved training practice. They also require the dentist to have weekly tutorials and complete a portfolio of experience. Foundation dentists attend 30 external study days during their 12-month long training programme. This is not a requirement for PLVE dentists, and it is widely perceived as a gap in the programme.

Starting in January 2020, the LDCs will provide a monthly study day which will include lectures and workshops, covering key elements of the portfolio, case discussion, peer review, audit, case presentation and support with portfolios.

For further information contact Philip Martin or Branan Sivanantha at LDCLeicester@aol.com.

\title{
At-home sleep bruxism assessment developed
}

Researchers from the

University of Eastern Finland have developed an easy-to-use electrode set to assess the severity of a patient's sleep bruxism in their own home. The technology was developed as part of a doctoral thesis project. ${ }^{1}$ The diagnostic accuracy was compared to that of conventional polysomnography and other bioelectric measurements.

Using the electrode set, nine out of ten at-home polysomnography recordings were of good quality, and the electrical impedance of the electrodes was at an

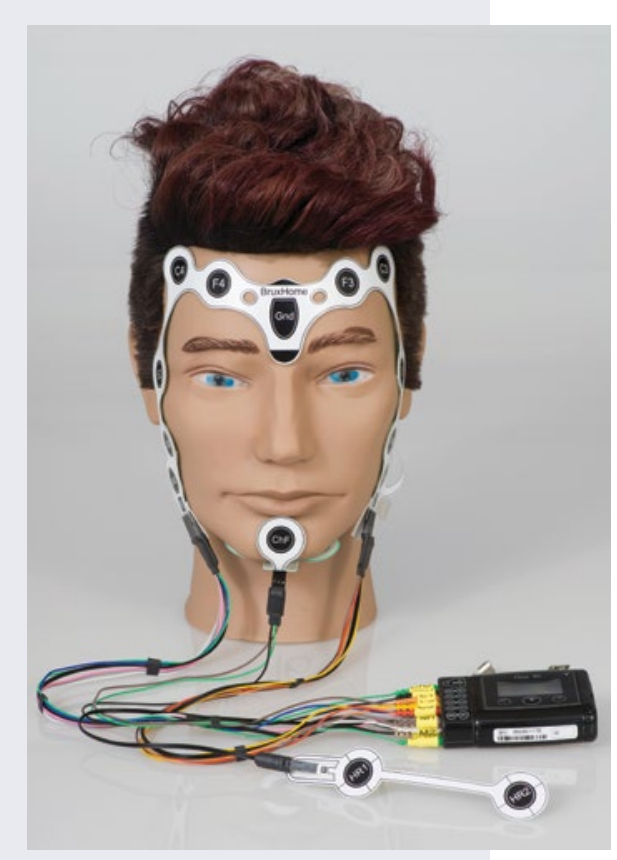

New polysomnography electrode set enables easy at-home assessment of sleep bruxism acceptable level $90 \%$ of the time. Only one out of 101 recordings failed due to problems related to the electrode set. Supporting evidence was also found for a previous finding that some patients may present altered results on the first night due to the so-called first night effect, and sleep bruxism assessment may require more than one night of recording.

\section{Reference}

1. Miettinen T. Suitability of a self-applicable electrode set for polysomnographic assessment of sleep bruxism in the home environment. 2019. Available at: http://epublications.uef.fi/pub/urn_isbn_978-952-61-3184-9/urn_isbn_978-952-61-3184-9.pdf (accessed October 2019).

\section{BDA AGM}

The BDA Western Counties Branch are holding their AGM on Monday 25 November 2019 at the Holiday Inn Express, Blackbrook Business Park, Blackbrook Park Avenue, Taunton, TA1 2PX. The AGM will start promptly at $7 \mathrm{pm}$. Following the AGM there will be a meal in the neighbouring 'Griffin' Harvester restaurant.

If you are able to attend the AGM, please email branchsectionevents@bda.org with your name and GDC number.

Several Branch Officer positions are open for election. For further details, or to stand for any of the Branch Officer positions, please contact Colin Silcox at westerncountiesbranch@ bda.org, ideally before Monday 18 November. 